\title{
Professionelle Bildverarbeitung auf einem PC
}

\author{
RICHARD A. BÖDI ${ }^{1}$ UND ThEODOR W. KAULICH ${ }^{2}$
}

Mathematisches Institut ${ }^{1}$ der Universität,
Abteilung für Medizinische Physik ${ }^{2}$ der Radiologischen Universitätsklinik, TÜBINGEN

\section{Zusammenfassung}

Der AMIGA 3000 Computer (Fa. Commodore) eignet sich hardwaremäBig für professionelle digitale Bildverarbeitung. Nach einer Darstellung der wesentlichen HardwareKomponenten werden Zusatzgeräte aus dem Video-Bereich vorgestellt. Es wird gezeigt, daB sich - zusammen mit den anschließend beschriebenen Bildverarbeitungs-Programmen - eine kostengünstige professionelle Bildverarbeitung auf einem $\mathrm{PC}$ realisieren läßt.

\section{Einleitung}

Professionelle Bildverarbeitung erforderte noch vor einiger Zeit zumindest mittelgroße Computer-Systeme; Computer aus dem PC- und Kleinrechner-Bereich besaßen einerseits zu wenig Speicher und andererseits war die Hardware zu leistungsschwach für solche Anwendungen. Die Leistungsgrenzen zwischen Workstations and PC's nähern sich jedoch immer mehr an. So gibt es mittlerweile auch leistungsstarke und dennoch kostengünstige Kleinrechner. Am Beispiel des AMIGA 3000 Computers wird gezeigt, daß nicht nur die Hardware dieses Rechners für digitale Bildverarbeitung und Video-Manipulation geeignet ist, sondern daß inzwischen auch die notwendige Software für professionelle Anwendungen zur Verfügung steht.

\section{Hardware des AMIGA 3000}

Der AMIGA 3000 wird mit einem $3 \frac{1}{2}$-Zoll FloppydiskLaufwerk, welches Disketten mit $880 \mathrm{kByte}$ formatiert, sowie einer SCSI-Quantum-Festplatte mit einer minimalen Größe von 52 MByte ausgeliefert. Das Betriebssystem ist in 512 kByte ROMs untergebracht; es kann bei Bedarf (etwa bei Updates) auch von der Festplatte oder von Diskette beim Boot-Vorgang geladen werden. Der RAM-Speicher umfaßt standardmäßig 2 MByte und kann intern ohne $\mathrm{Zu}$ satzkarten auf maximal $18 \mathrm{MBy}$ te erweitert werden, wobei bis zu 2 MByte RAM als Vidco-Speicher dienen können.
Der AMIGA 3000 basiert auf einer mit $25 \mathrm{MHz}$ getakteten Motorola 68030 CPU und einem Coprozessor des Typs MC68882, der ebenfalls mit $25 \mathrm{MHz}$ betrieben wird. $\mathrm{Zu}$ sätzlich besitzt der A3000 zwei weitere, frei programmierbare Coprozessoren, die COPPER und BLITTER genannt werden. Während der COPPER für die Darstellung des Videobildes zuständig ist, läßt sich der BLITTER sowohl zum Kopieren und Verknüpfen von bis zu vier verschiedenen Speicherbereichen, als auch zum Linienziehen und zum Flächenfüllen verwenden.

Trotz der geringen Gehäuseabmessungen können bis zu vier Erweiterungskarten und eine zusätzliche Prozessorkarte in den AMIGA 3000 eingebaut werden. Durch die offene Systemarchitektur (OSA) können nicht nur Speichererweiterungen und schnelle Prozessorkarten (verschiedene Turbokarten mit der Motorola CPU 68040 sind derzeit erhältlich) zur Performance-Steigerung eingesetzt werden. Ebenso können mit minimalem Aufwand Graphik- und Videokarten (z.B. Framebuffer, Digitizer oder die Video-Multifunktionskarte VideoToaster, Fa. NewTek, Topeka, USA) zur Manipulation von digitalen Bildern und von VideoSignalen in den Rechner integriert werden. Ein derart aufgerüsteter $A M I G A$-Rechner kann dadurch oftmals eine wesentlich teurere Video-Anlage ersetzen. Vereinfacht wird der Einsatz des AMIGA im Video-Bereich dadurch, daß das standardmäßig verwendete Videosignal des A3000 wahlweise in PAL oder NTSC vorliegt, wobei Video-Anschlüsse für $15 \mathrm{kHz}$ und $31 \mathrm{kHz}$ vorhanden sind. Mit Hilfe eines Genlocks lassen sich sehr kostengünstig beliebige Videoquellen anschließen und auf dem Computer-Bildschirm anzeigen. Dabei kann das externe Videosignal auf vielfältige Weise mit dem Videosignal des Rechners gemischt werden. So läßt sich nicht nur der Hintergrund des Bildschirmes durch das externe Videosignal ersetzen, sondern auch jede beliebige andere Farbe. Mit Hilfe eines Digitizers, z.B. dem VLab (Fa. Macro Systems, Witten), lassen sich die gemischten Bilder dann in Echtzeit (25 Vollbilder pro Sekunde) digitalisieren. Der VLab Digitizer zeichnet sich dabei durch gute Bildqualität (in 24 Bit) bei schr günstigem Preis (ca. DM 600.-) aus. Mit VLab lassen sich Bilder sogar direkt auf 
die Festplatte digitalisieren (ca 1.5 Sekunden bei $750 \times 580$ Pixel und einer Quantum-Festplatte). Frgänzt wird dieser Digitizer durch die umfangreiche mitgelieferte Software, die in beschränktem Maße nogar cine Bildnachbearbeitung zuläßt.

\section{Software fir professionclle Bildverarbeitung}

Mittlerweile gibt es für den AMIGA einige Programme zur digitalen Bildverarbeitung. Die für den professionellen Einsatz übliche Farbtiefe digitaler Bilder beträgt 24 Bit. Bilder dieser Farbtiefe konnten mit den bislang für den AMIGA erhältlichen Programmen nicht bearbeitet werden. Mit dem Erscheinen der Programme Art Department Professional (Fa. ASDG, Madison, USA) und Image Master (Fa. Black Belt Systems) hat sich diese Situation jedoch grundlegend geändert. Beide Programme führen sämtliche Operation in 24 Bit Farbtiefe durch. Bilder geringerer Farbtiefe werden auf das 24 Bit Format umgerechnet. Das Programm Art Department Professional (ADP) ist modular aufgebaut. Zusätzliche Funktionsmodule werden von ASDG fortlaufend entwickelt und angeboten. Die Bilder können entweder über eine 24 Bit Graphikkarte direkt angezeigt oder nach einer geeigneten Transformation ohne Hardware-Zusätze in einem Anzeige-Format des AMIGA dargestellt werden. Das Programm ADP unterstützt eine Vielzahl verschiedener Graphik-Formaten wie z.B. IFF-ILBM, TIFF, GIF, JPEG, PCX, PostScript, MacPaint und SunRaster. Dadurch eignet sich ADP auch als Konvertierungsprogramm. Beim Laden von Bildern können diese auch miteinander gemischt werden, wobei das Mischungsverhältnis zwischen zwei Bildern frei eingestellt werden kann. Unter den Lade-Operationen befinden sich auch Routinen zum Erzeugen von Farbverläufen, die sich z.B. als Bild-Hintergrund eignen. Die Art des Farbverlaufes und die Anzahl der dabei verwendeten Farben kann flexibel gewählt werden. Im Programmteil "Colors Control" lassen sich die Farbanteile von Rot, Grün und Blau, die Helligkeit und der Kontrast, sowie der Grad einer Gamma-Korrektur fest- legen. Darüberhinaus können Dithering-Verfahren verwendet werden, um z.B. bei Bildschirm-Darstellungen mit geringer Farbanzahl glattere Farbverläufe zu erhalten. Bislang verfïgt $A D P$ über fïnf verschiedene Dithering-Algorithmen, darunter die Verfahren von Floyd-Steinberg, Burkes und Jarvis. Mit IIilfe der Palette-Funktion ist es möglich, einzelne Farben oder auch Farbgruppen in einem komfortablen Editor vielfältig zu bearbeiten. Den umfangreichsten Teil von ADP stellen die zahlreichen Filter und Transformationen dar, die sich auf die Bilder anwenden lassen. Diejenigen Filter, die über eine Matrix definierbar sind, können frei definiert, geladen und abgespeichert werden. Etwa 25 Filter-Matrizen sind schon vordefiniert. Mit Hilfe des Frame-Editors können sämtliche Operationen auf ganze Bildserien angewendet werden. Für die meisten Funktionen benötigt das Programm nur wenige Sekunden. Über alle bisher beschriebenen Funktionen verfügt auch das Programm Image Master. Zusätzlich bietet Image Master umfangreiche 24 Bit Malfunktionen, die Erzeugung und Verwaltung von 24 Bit Animationen, sowie zahlreiche Spezialeffekte. Außerdem können alle Operationen auf frei definierbare Bildsegmente eingeschränkt werden und sind zudem in ihrer Intensität regelbar.

\section{Diskussion}

Vergleicht man die Leistungen, die man bei der Kombination von AMIGA 3000 mit Video-Hardware und Bildverarbeitungs-Software erhält, mit den Kosten dieser Konfiguration (ca. DM 6000.-), so ergibt sich ein Preis-LeistungsVerhältnis, das bei anderen Rechner-Systemen bisher nicht erreicht wird. Dies ist vor allem durch die auf dem PALSystem beruhende Graphik des AMIGA begründet. Dadurch entfallen nämlich umfangreiche und teuere InterfaceSchaltungen, die bei anderen Rechner-Systemen nötig sind. Hinzu kommt der günstige Preis (ca. DM 500.- bis DM 1000.-) der Bildverarbeitungs-Software, die für den professionellen Einsatz geeignet ist. 